\title{
BMJ Open Native American patients' perception and attitude about kidney transplant: a qualitative assessment of patients presenting for kidney transplant evaluation
}

Mira Keddis, ${ }^{1}$ Dawn Finnie, ${ }^{2}$ Wonsun (Sunny) Kim ${ }^{3}$

To cite: Keddis M, Finnie D, Kim W(Sunny). Native American patients' perception and attitude about kidney transplant: a qualitative assessment of patients presenting for kidney transplant evaluation. BMJ Open 2019;9:e024671. doi:10.1136/ bmjopen-2018-024671

- Prepublication history and additional material for this paper are available online. To view please visit the journal (http:// dx.doi.org/10.1136/bmjopen2018-024671).

Received 22 June 2018 Revised 24 December 2018 Accepted 27 December 2018

Check for updates

(c) Author(s) (or their employer(s)) 2019. Re-use permitted under CC BY-NC. No commercial re-use. See rights and permissions. Published by BMJ.

${ }^{1}$ Division of Nephrology and Hypertension, Department of Medicine, Mayo Clinic, Scottsdale, Arizona, USA ${ }^{2}$ Center of Science of Health Care Delivery, Mayo Clinic, Rochester, Minnesota, USA

${ }^{3}$ College of Nursing and Health Innovation, Arizona State University, Phoenix, Arizona, USA

Correspondence to

Dr Mira Keddis;

keddis.mira@mayo.edu

\section{ABSTRACT}

Objective Native Americans suffer from lower rates of kidney transplantation compared with whites. Our goal was to elicit patients' perceptions of and attitudes about kidney transplant and the impact of financial burden and cultural taboos.

Design This is an exploratory qualitative interview study of 12 Native American patients recruited after completion of the kidney transplant evaluation.

Setting Semistructured interviews were conducted. Interviews were coded using inductive methods, followed by interpretive coding by the investigators.

Results Thematic analysis revealed the following themes: (1) experience with kidney transplant education by the healthcare team; (2) cultural beliefs regarding kidney transplant; (3) personal motivation and attitude towards kidney transplant; (4) financial burden of kidney transplant and post-transplant care and (5) attitude about living donation. Most participants were educated about transplant as a treatment option after dialysis initiation. All patients in this study recognised that some taboos exist about the process of organ procurement and transplantation; however, the traditional views did not negatively impact their decision to pursue kidney transplant evaluation. Patients shared the common theme of preferring an organ from a living rather than a deceased person; however, the majority did not have a living donor and preferred not to receive an organ from a family member. Most patients did not perceive transplant-related cost as negatively impacting their attitude about receiving a transplant even for patients with below poverty level income.

Conclusions Native American patients presenting for kidney transplant were less likely to be educated about transplant before dialysis initiation; did not perceive financial burden and cultural beliefs were not discussed as obstacles to transplant. While a living donor was the preferred option, enthusiasm for living donation from family members was limited.

\section{BACKGROUND}

Native American (NA) patients have higher rates of end-stage renal disease (ESRD)
Strengths and limitations of this study

- This is the first qualitative study of 12 full-descendant Native Americans with end-stage renal disease presenting for kidney transplant evaluation.

- The patients interviewed may have presented themselves in a more positive way with fear that their input may influence the outcome of the transplant evaluation.

- The findings of this study may not be generalisable to other cohorts of Native Americans with kidney disease prior to transplant evaluation.

compared with whites, attributed to higher rates of diabetes. ${ }^{1-3}$ Compared with dialysis, kidney transplantation is considered the preferred treatment of choice for select candidates with end-stage kidney disease with evidence supporting improved longterm survival, ${ }^{4-7}$ quality of life ${ }^{8}$ and decreased cost. ${ }^{9}{ }^{10}$ However, rates of waitlisting on the United Network for Organ Sharing (UNOS) and rates of kidney transplantation are significantly lower for NA patients compared with other minority groups and whites. ${ }^{11-13}$

Studies examining determinants of the inequity in the rates of waitlisting and kidney transplantation show several contributing clinical and socioeconomic variables. For example, the prevalence of blood type $\mathrm{O}$, diabetes and dialysis is higher for NA patients, and these factors are associated with lower rates of kidney transplantation. ${ }^{11121415}$ Of the socioeconomic factors, poverty level income and government insurance significantly contribute to the lower rates of transplant for NA patients. ${ }^{11121415}$ These factors however do not completely explain the lower rates of waitlisting and transplantation for NA compared with whites. This suggests that unmeasured variables may be playing a role. 
Black Americans share similar rates of waitlisting and kidney transplantation as NA patients. ${ }^{11}{ }^{12}$ Several qualitative studies of black Americans with kidney disease have been performed to identify patients' perspectives and attitudes about kidney transplantation. ${ }^{16}$ Focus group discussion of 36 black dialysis patients showed concerns about poor treatment by dialysis technicians, lack of knowledge about kidney transplantation and fear of surgery as factors impacting patients' attitude about kidney disease and transplant. ${ }^{17}$ Another study of 33 black patients presenting for kidney transplant evaluation showed that lack of knowledge and cultural factors such as perceived discrimination and mistrust of the healthcare system were independently predictive of delays in being accepted as kidney transplant candidates. ${ }^{18}$ Similar qualitative studies of NA patients with kidney disease are lacking.

Qualitative studies of NA patients investigating attitudes to organ donation and transplantation have suggested several important themes that may negatively impact their attitude about kidney transplant, similar to those identified for black patients. These include: fear of surgery, belief that the body must remain whole to enter the spirit world, doubt of the ethical process of organ procurement and mistrust of the safety and quality of deceased organs. ${ }^{19-23}$ These findings however were from qualitative studies of patients without kidney disease.

The objective of this exploratory, qualitative study is to assess the perceptions of and attitudes about kidney transplant in a cohort of NA patients with kidney disease undergoing kidney transplant evaluation at Mayo Clinic Arizona.

\section{METHODS}

\section{Study design and setting}

Arizona is in ESRD Network 15 where NA patients account for $11.5 \%$ of prevalent dialysis patients, which is the highest percentage of NA patients on dialysis in the USA. However, NA patients represent only $5.1 \%$ of prevalent kidney transplant patients in Network $15 .^{24}$ Mayo Clinic Arizona serves as a referral centre for kidney transplant for Network 15 with $>300$ kidney transplant evaluations per year and an average of 30 NA patients receiving kidney transplant per year over the last 5 years. Qualitative analysis using grounded theory method and inductive thematic analysis ${ }^{25} 26$ was used to identify emergent themes from transcripts of interviews with NA patients presenting for kidney transplant evaluation.

\section{Interview guide preparation}

A semistructured interview guide was based on topics existing in the literature and was structured to elicit information about the patient's knowledge about transplant as a treatment option for their disease, the perceived burden of kidney disease and dialysis as it pertains to their social, financial and family well-being and the impact of that on their motivation for a kidney transplant, the influence of their cultural beliefs on their attitude about receiving a kidney transplant and attitude and motivation for kidney transplant. A copy of the interview guide is included in online supplemental material. The goals and objectives of the study were outlined to the patients by the interviewer at the beginning of the interview process.

\section{Participant selection}

Patients were recruited using the transplant database. All patients scheduled for a kidney transplant evaluation were identified and screened in a consecutive pattern. Of those, patients who self-identified as NA/Alaskan Native were screened for inclusion in the study. The inclusion criteria included (1) chronic kidney disease with estimated glomerular filtration rate less than $20 \mathrm{~mL} /$ $\min / 1.73 \mathrm{~m}^{2}$ adjusted body surface area using the Modification of Diet in Renal Disease estimating equation or on dialysis and (2) non-Hispanic or Latino race. A research coordinator met with the patients to determine their interest in participating in the study. Patients who consented to the study were contacted within 2 weeks after the initial consent. Those who provided written informed consent and participated in the interview were remunerated. The study period extended from February to October 2017, and the study was closed for recruitment after determination of theoretical saturation by the study investigators. Theoretical saturation was based on review of the interview transcripts and noting similar repeated responses allowing for theme development and low likelihood that more interviews would provide added information to generate new categories or themes.

\section{Chart abstraction data collection}

The following baseline characteristics were abstracted from the medical record: age, gender, dialysis requiring and presence of diabetes, cause of ESRD and prior history of kidney transplant evaluation. We abstracted the following socioeconomic variables from the social worker database: education level, annual household income, insurance type and distance from the transplant centre and employment status. Psychosocial factors analysed included tribe affiliation, marital status and caregiver plan.

\section{Interview data collection and analysis}

A semistructured interview guide was designed by interviewer (MK) and underwent a peer review process for improvements. The questions were designed to explore patient's perceptions of their kidney disease and attitude and motivation for kidney transplant. Other questions addressed influence of patients' and their families cultural and religious beliefs surrounding kidney transplantation and the role these beliefs and values played in the decision making process. The interviewer (MK) was influenced by her understanding of kidney disease and kidney transplant and consequently, the interviewer influenced the interview by the semistructured nature of the interview guide as well as her understanding in the field. The shared understanding of the kidney transplant 
between the interviewer and the patients being interviewed allowed the interviewer to be an active participant in both the interview and analysis. The interviewer did not have an established relationship with the study participants prior to the start of this study.

\section{Patient and public involvement}

The initial interview guide was constructed based on established knowledge from qualitative and quantitative literature on factors contributing to reduced rates of waitlisting and kidney transplantation and NA views and perceptions of donation and kidney transplantation. ${ }^{2311} 1319202223$ The interview guide was continually improved throughout the study by incorporating implicit input from the patients interviewed. Patients were offered the opportunity to review the results of the study on completion.

\section{Data analysis}

All interviews were audio recorded, transcribed, and the audio recordings were also used so the analysts could consider inflection and emotion of the participants. The data were analysed using grounded theory and inductive qualitative thematic analysis. ${ }^{27}$ An iterative cycle of interviews, data collection and analysis was performed. After the first two interviews, the transcripts were reviewed, and the interview questions were modified. This iterative cycle continued after every four interviews thereafter.

\section{Coding process}

The analysis team (MK, DF) independently read the transcripts of the first two interviews and began developing codes inductively based on the data. ${ }^{27}$ Once a codebook was constructed, each analyst read the interviews again and independently applied the codes that had emerged. The analysis team then met and discussed the coded transcripts to ensure consensus of each coded passage. The coding process was applied to each subsequent interview. Additional codes were added where appropriate and codes were redefined during this iterative analysis process.

Main themes were given priority in subsequent analysis and those themes are presented in this manuscript. The research question, purpose of this study and uniqueness of the themes that emerged contributed to guiding our selection of themes to prioritise.

\section{RESULTS}

\section{Participant characteristics}

Over a 9-month recruitment period, a total of 260 patients underwent kidney transplant evaluation, 38 patients self-identified their race as American Indian/Alaskan Native and a total of 23 patients consented. Twelve patients completed the interview. Four interviews were completed in person in the outpatient transplant clinic setting and interview duration ranged from 26:41 (min:s) to $57: 58$ (min:s). Two of the patients who completed the interview in person had one family member present for the duration of the interview. All patients identified themselves to be of NA descent from both parents, 6 were affiliated with the Navajo tribe and 10 lived on the reservation. There were seven male, and the mean age was 50.1 $( \pm 8.65)$ years. Seven were initiated on dialysis prior to kidney transplant evaluation and nine had diabetes and diabetic kidney disease.

Ten of the 12 patients had high school or graduate level education. Five patients reported that cultural beliefs were the primary source of their religious affiliation. All but one had an identifiable caregiver. Only one had a prior kidney transplant evaluation at Mayo Clinic.

\section{Patient perceptions about kidney transplant}

Five major themes emerged from the data: (1) kidney transplant education by the healthcare team; (2) kidney transplant and cultural beliefs; (3) motivation and attitude towards kidney transplant; (4) financial burden of kidney transplant and post-transplant care and (5) attitude about living donation.

\section{Kidney transplant education by the healthcare team prior to transplant evaluation}

NA patients with kidney disease received care both within and outside the Indian Health Services (IHS). They reported that education about kidney transplant as a treatment option for kidney disease took place at the time of dialysis initiation or months to years later. Five patients were referred to transplant before dialysis initiation. Only one patient shared that their provider emphasised transplant as a better treatment option compared with dialysis.

Supporting quotes for theme 1: transplant as a treatment option for kidney disease

Patient 1:'getting orientation to get into the DaVita dialysis was the first time I heard about 'transplant' as part of the orientation.'

Patient 6:'My doctor didn't mention much about transplant but later on once I started on dialysis...he talked me about transplant.'

Patient 10:'About twoyears after my dialysis treatments.'

\section{Kidney transplant and cultural taboos}

All NA patients in this study recognised that some cultural taboos exist about the process of organ procurement and transplantation; however, these taboos did not negatively impact their decision to pursue kidney transplant evaluation. Patients shared the common theme of preferring an organ from a living rather than a deceased person. Patients who described themselves as religious reported the positive role of prayer.

Supporting quotes for theme 2: cultural beliefs and the decision to proceed with kidney transplant evaluation

Patient 2: 'If you take an implant from a dead person, like a dead person's kidney it will put a curse on you or it will never work for you or if you get a kidney 
from a white guy or black guy or Mexican guy it will not work for your body, it will shut down. That's what they think. ... They talk to you about ghost things that the ghost of the person who gave you the life is going to haunt you...I just don't think about it and if they give me one like that, I don't care... I believe 'this' but it's not their lives it's my life.'

Patient 3:'Every Native American culture kinda frowns upon the fact that you are getting a kidney from a cadaver...For me it is a lifesaving procedure. It is giving you your life back and I'm very good if I get a cadaver.'

Patient 5:'It is kinda taboo to take any person's body part and put them in yours. ...A lot of elders don't approve of it...why do you want to bring any kind of weirdness into your family, evil kind of thing, but I guess to me my quality of life, traditions or no traditions I'd rather have something to live for.'

\section{Motivation and attitude towards kidney transplant}

Patients unanimously expressed that their family and loved ones were the primary motivation to pursue a kidney transplant. The second most common reason was avoidance or liberation from dialysis and improved quality of life. Patients also expressed desire to resume social activity and employment after transplant.

Patients were asked to reflect on what their feelings would be if they were not able to receive a kidney transplant for any reason. Patients responded with positive sentiments that expressed acceptance of the future. Positive thinking and resiliency regarding overcoming their diagnosis of kidney disease and dialysis for some enabled them to be patient and flexible regardless of the circumstances. One patient expressed an attitude of disappointment and fear for their family.

Supporting quotes for theme 3 : motivation and attitude towards kidney transplant

\section{Motivation for transplant}

Patient 1: 'my hope to live for my loves ones... I love to hold my grandkids.'

Patient 3: 'I'm tired of being sick. I don't want to be tied down for 4 hours three times a week.'

Patient 10: 'my son. He wants me here a little bit longer.'

\section{Attitude if they were not able to receive a kidney transplant}

Patient 1:'if something doesn't work it's not going to bring me down and it's not going to yank the bottom of my little world like this sickness did...I'm a patient person and I'll just take it out and roll with it as it comes and mature.'

Patient 4:'I don't want to be hurt by that or get stressed about it... I want to be way up there and keep my self-going in life.'

Patient 11:'we don't make our plans for ourselves: we're just on this journey'
Table 1 Patients' perception of financial burden of kidney transplant with respect to their insurance coverage, employment status and annual income

\begin{tabular}{llc}
\hline Patient & $\begin{array}{l}\text { Perceived financial } \\
\text { burden }\end{array}$ & $\begin{array}{l}\text { Annual household } \\
\text { income }(\mathbf{\$})\end{array}$ \\
\hline 1 & Yes & 32400 \\
2 & No & 26256 \\
3 & No & 42000 \\
\hline 4 & Yes & 41220 \\
\hline 5 & Yes & 54000 \\
6 & No & 15132 \\
\hline 7 & Yes & 48000 \\
\hline 8 & No & 15600 \\
\hline 9 & No & 16200 \\
\hline 10 & No & 18000 \\
\hline 11 & No & 106800 \\
\hline 12 & Yes & 120000 \\
\hline
\end{tabular}

Patient 12:'I would feel sad and upset... I would think of my kids and how they would have to carry on without their mother.'

\section{Financial burden of kidney transplant and post-transplant care}

Patient's attitude about finances as potential barrier to undergoing kidney transplant and for post-transplant care varied widely. Although, five patients expressed some concern; the others expressed confidence in their insurance and either the reservation or Indian Health to provide needed funds. The majority had private insurance $(66.7 \%)$ and four were employed. Median income of the group was US\$36810 but three patients $(6,8,9)$ had income below poverty level ( $\leq$ US $\$ 16240)$ as shown in table 1. It is of note that these three patients did not feel that the cost of transplant and related medications would hinder them from pursuing a kidney transplant. Patients who expressed concern about finances shared plans to improve their income to help facilitate needed cost.

Supporting quotes for theme 4: financial burden of kidney transplant and post-transplant care

Patient 11:'I have all the medical insurance and I have backup from IHS.'

Patient 3: 'I am thoroughly prepared to take care of my portion of what I need to do and for me you cannot put a price tag on life.'

Patient 4:"Between me and my daughter we are planning to start making money and....start saving.'

\section{Attitude about living donation}

Patients in this cohort were comfortable with the concept of receiving a living donor and preferred a living donor over a deceased donor. Only two patients in the cohort reported having a living donor. Ten patients did not have 
a living donor and would not consider a family member as a donor for several reasons including: lack of knowledge about this process, uncertainty about the quality of the kidney based on the lifestyle of the donors, desire not to burden family members and mistrust of the intentions of family members who volunteered to donate.

\section{Supporting quotes for theme 5: attitude about living donation}

Patient 6: 'my kids and my relatives but I don't want to depend on them. I want them to live their own lives... I don't mind delaying getting a kidney whenever that will be.'

Patient 7: 'there are people willing but I've made a choice not to take anything from anybody I know.'

Patient 8: 'my daughter wanted to but I'd rather her enjoy it with her kids.'

Patient 11: 'to be honest, I would prefer a living organ.'

\section{DISCUSSION}

To our knowledge, this is the first qualitative study of NA patients (full NA descendants) with kidney disease presenting for kidney transplant evaluation to explore their perspectives of and attitudes about receiving a kidney transplant. The results highlight important themes that influence the patients' perceptions about kidney transplant, some of which may contribute to delays and lower rates of kidney transplantation. Knowledge of transplant as a treatment option for kidney disease was commonly delayed until after dialysis initiation for the majority of patients. This is consistent with studies showing that NA patients were more likely to require dialysis before transplant compared with whites. ${ }^{11}{ }^{13}$ Despite evidence for detrimental effects of dialysis on post-transplant outcomes especially among diabetics, ${ }^{28}$ efforts at improving the healthcare systems' process of timely transplant education have not yet achieved the desired goal. The majority of the patients received care at the IHS, and this provides an opportunity for focused intervention to improve timely transplant education.

NAs recognise that cultural taboos may negatively influence their receptiveness to consider kidney transplantation as a treatment for kidney disease. Our patient cohort's motivation for transplant for the sake of their families and their loved ones transcended their knowledge and/or fears of these taboos. This is in contrast to other qualitative analyses highlighting the reluctance to participation in donor and transplant programmes due to culturally determined attitudes. ${ }^{19-22}$ This discrepancy can be explained by two factors: first, studies of NA individuals and transplantation did not include patients with kidney disease and/or on dialysis. Second, our cohort is biased towards a unique group of NA patients who agreed to a transplant referral and presented for their appointments. It should be noted however that NA individuals have reported that they would respect their loved one's decision to donate organs even if that decision is in opposition to cultural beliefs. ${ }^{22}$ This again highlights the importance of family for NA patients. We hypothesise that the negative implications of cultural taboos towards transplant are influenced by patients' age, family support and their stage in the kidney transplant process. This needs further testing in mixed-method analytical approaches.

Attitude and motivation for transplant among this cohort of NA patients differed from other populations. All NA patients in this cohort emphasised the primary goal of pursuing a kidney transplant was to be present and care for their loved ones followed by avoidance of dialysis and its associated burden of physical symptoms and time commitment. In a cohort of Moroccan dialysis-dependent patients, the primary motivation for transplant was to be liberated from dietary and fluid constraints. ${ }^{29}$ For patients with ESRD in China, the most commonly reported reason for pursing a kidney transplant was to have freedom to study and continue to work. ${ }^{30}$ The findings of our study emphasise the important role of families for NA patients. Incorporating family members in transplant education prior to transplant referral may positively impact access to transplant for NA patients. The reaction to the possibility of not receiving a kidney transplant in our cohort illustrated how NA patients perceived the transplant process and its outcome as reflective of their fate and as such all but one patient expressed acceptance of the outcome. How this attitude influences NA patient's commitment to pursuing kidney transplant requires further study.

NA patients suffer from socioeconomic disparity compared with whites. ${ }^{11} 13$ Quantitative studies have shown that poverty explained some of the disparity that accounted for lower rates of waitlisting after transplant evaluation for NA patients. Our findings are first to illustrate that most NA patients undergoing kidney transplant evaluation do not perceive the financial cost of kidney transplant and post-transplant care as a barrier to pursuing a kidney transplant. A striking observation is the discrepancy between patient's reported incomes as documented by the social worker record and the patient's perception of financial burden. Our findings therefore argue that financial burdens may not be a perceived barrier to presenting for kidney transplant evaluation for NA patients.

The attitude towards living donation by our cohort is reflective of the known lower rates of living donor transplant for NA patients compared with whites. ${ }^{11}$ Our findings are consistent with the literature showing that NA patients prefer living over deceased kidney transplant. ${ }^{19-21}$ Reluctance to consider family members as potential donors has been shown in other cohorts and not unique to NA patients. ${ }^{31}$ Our NA cohort's primary reasons in not pursuing discussions or consideration for living donation from family or friends were not thinking about that as an option and to prevent an added burden to their loved ones. Others have shown that some of the reasons NA patients refused to donate organs included not considering that as an option and a desire for the body to stay 
whole. ${ }^{22}$ Patient and family educational efforts to improve knowledge of the living organ donation process have been trialled, and efforts are needed to address this gap in knowledge.

Several limitations warrant discussion. First this qualitative study was reflective of an in-depth interview of 12 full-descendant NA patients. While the number of patients' interviewed is small, this study is first to assess perception, motivation and attitude about kidney transplant in a cohort of NA patients with kidney disease and provides insights that focus the emphasis on interventions that may promote increased acceptance of kidney transplantation including early education, family involvement and less emphasis on financial burden. Only $52 \%$ of those who consented completed the interview and this was due to several factors including: unwillingness to commit the time for the interview, the need to avoid delays in travelling back to their home after completion of their appointments, patients not answering or returning phone calls and language barriers. Second, the findings reflect the input of patients who presented for evaluation and may not be reflective of patients who have not yet agreed to pursue a kidney transplant referral and evaluation. Third, the results of the interviews may have been biased in that patients may have presented themselves in a more positive way with the fear that their opinions may influence the transplant candidacy process. Future studies engaging representative NA patients and NA healers in the interview process are necessary.

\section{CONCLUSIONS}

Our study emphasises the role of early transplant education and family involvement as modifiable factors that may help to increase acceptance of kidney transplantation as a treatment option for kidney disease for NA patients and highlights the disconnect between reported and perceived financial burden of kidney transplantation. Future studies of NA patients at different stages of the kidney transplant evaluation process may guide interventions to improve rates of waitlisting and kidney transplantation.

Acknowledgements We thank our patients who invested their time to share their valuable and honest input which made this work possible.

Contributors MTK, WK: research idea and study design. MTK: data acquisition. MTK, WK, DF: data analysis/interpretation. MTK, DF: data interpretation. WK, DF: supervision or mentorship. Each author contributed important intellectual content during manuscript drafting or revision and accepts accountability for the overall work by ensuring that questions pertaining to the accuracy or integrity of any portion of the work are appropriately investigated and resolved.

Funding This work was supported by an institutional grant through the Office of Health Care Disparity at Mayo Clinic.

Disclaimer MTK affirms that the manuscript is an honest, accurate and transparent account of the study being reported; that no important aspects of the study have been omitted and that any discrepancies from the study as planned have been explained.

Competing interests None declared.

Patient consent for publication Not required.
Ethics approval Mayo clinic institutional review board approved the study.

Provenance and peer review Not commissioned; externally peer reviewed.

Data sharing statement There are no additional data available. The data are accessible by the principal investigator of this study MK. The data is summarised in this paper. The deidentified transcribed patient interviews can be provided by MK upon request.

Open access This is an open access article distributed in accordance with the Creative Commons Attribution Non Commercial (CC BY-NC 4.0) license, which permits others to distribute, remix, adapt, build upon this work non-commercially, and license their derivative works on different terms, provided the original work is properly cited, appropriate credit is given, any changes made indicated, and the use is non-commercial. See: http://creativecommons.org/licenses/by-nc/4.0/.

\section{REFERENCES}

1. United States Renal Data System. 2016 annual data report: atlas of chronic kidney disease and end-stage renal disease in the United States, National Institutes of Health, National Institute of Diabetes and Digestive and Kidney Diseases. 2016.

2. Narva A, Stiles S, Karp S, et al. Access of Native Americans to renal transplantation in Arizona and New Mexico. Blood Purif 1996;14:293-304.

3. Narva AS. Kidney disease in Native Americans. J Natl Med Assoc 2002;94:738-42.

4. Wolfe RA, Ashby VB, Milford EL, et al. Comparison of mortality in all patients on dialysis, patients on dialysis awaiting transplantation, and recipients of a first cadaveric transplant. N Engl $J$ Med 1999;341:1725-30.

5. Schnuelle P, Lorenz D, Trede M, et al. Impact of renal cadaveric transplantation on survival in end-stage renal failure: evidence for reduced mortality risk compared with hemodialysis during long-term follow-up. J Am Soc Nephrol 1998;9:2135-41.

6. Port FK, Wolfe RA, Mauger EA, et al. Comparison of survival probabilities for dialysis patients vs cadaveric renal transplant recipients. JAMA 1993;270:1339-43.

7. Meier-Kriesche HU, Ojo AO, Port FK, et al. Survival improvement among patients with end-stage renal disease: trends over time for transplant recipients and wait-listed patients. J Am Soc Nephrol 2001;12:1293-6.

8. Kostro JZ, Hellmann A, Kobiela J, et al. Quality of life after kidney transplantation: a prospective study. Transplant Proc 2016;48:50-4.

9. Laupacis A, Keown P, Pus N, et al. A study of the quality of life and cost-utility of renal transplantation. Kidney Int 1996;50:235-42.

10. Loubeau PR, Loubeau JM, Jantzen R. The economics of kidney transplantation versus hemodialysis. Prog Transplant 2001;11:291-7.

11. Hall YN, Choi Al, Xu P, et al. Racial ethnic differences in rates and determinants of deceased donor kidney transplantation. J Am Soc Nephrol 2011;22:743-51.

12. Joshi S, Gaynor JJ, Bayers S, et al. Disparities among Blacks, Hispanics, and Whites in time from starting dialysis to kidney transplant waitlisting. Transplantation 2013;95:309-18.

13. Sequist TD, Narva AS, Stiles SK, et al. Access to renal transplantation among American Indians and Hispanics. Am J Kidney Dis 2004; 44:344-52.

14. Schold JD, Gregg JA, Harman JS, et al. Barriers to evaluation and wait listing for kidney transplantation. Clin J Am Soc Nephrol 2011;6:1760-7.

15. Keith D, Ashby VB, Port FK, et al. Insurance type and minority status associated with large disparities in prelisting dialysis among candidates for kidney transplantation. Clin J Am Soc Nephrol 2008;3:463-70.

16. Harding K, Mersha TB, Pham PT, et al. Health disparities in kidney transplantation for African Americans. Am J Nephrol 2017;46:165-75.

17. Salter ML, Kumar K, Law AH, et al. Perceptions about hemodialysis and transplantation among African American adults with endstage renal disease: inferences from focus groups. BMC Nephrol 2015;16:49.

18. Myaskovsky L, Almario Doebler D, Posluszny DM, et al. Perceived discrimination predicts longer time to be accepted for kidney transplant. Transplantation 2012;93:423-9.

19. Newton JD. How does the general public view posthumous organ donation? A meta-synthesis of the qualitative literature. BMC Public Health 2011;11:791.

20. Danielson BL, LaPree AJ, Odland MD, et al. Attitudes and beliefs concerning organ donation among Native Americans in the upper Midwest. J Transp/ Coord 1998;8:153-6. 
21. Filippi MK, Young KL, Nazir N, et al. American Indian/Alaska Native willingness to provide biological samples for research purposes. $J$ Community Health 2012;37:701-5.

22. Fahrenwald NL, Belitz C, Keckler A. "Tribes sharing life": an organ donation educational intervention for American Indian tribal college and university students. West J Nurs Res 2011;33:901-15.

23. Fahrenwald NL, Stabnow W. Sociocultural perspective on organ and tissue donation among reservation-dwelling American Indian adults. Ethn Health 2005;10:341-54.

24. Saran R, Li Y, Robinson B, et al. US Renal Data System 2015 Annual Data Report: epidemiology of kidney disease in the United States. Am J Kidney Dis 2016;67(3 Suppl 1):A7-A8.

25. Glaser BGS, A.L.; Strutzel E. The discovery of grounded theory. Nursing Research 1968;17:364-5.

26. Glaser BG, Strauss AL. The purpose and credibility of qualitative research. Nurs Res 1966;15:56-61.
27. Green JaT N. Qualitative methods for health research: Sage Publications, 2014

28. Cosio FG, Hickson LJ, Griffin MD, et al. Patient survival and cardiovascular risk after kidney transplantation: the challenge of diabetes. Am J Transplant 2008;8:593-9.

29. Kabbali N, Mikou S, El Bardai G, et al. Attitude of hemodialysis patients toward renal transplantation: a Moroccan Interregional Survey. Transplant Proc 2014;46:1328-31.

30. Qiao B, Liu L, Liu J, et al. A study on the attitude toward kidney transplantation and factors among hemodialysis patients in China. Transplant Proc 2016;48:2601-7.

31. Calestani M, Tonkin-Crine S, Pruthi R, et al. Patient attitudes towards kidney transplant listing: qualitative findings from the ATTOM study. Nephrol Dial Transplant 2014;29:2144-50. 